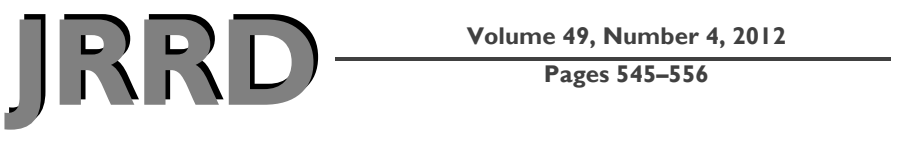

\title{
Initial developmental process of a VA semistructured clinical interview for TBI identification
}

\author{
Rodney D. Vanderploeg, PhD; ${ }^{1-5 *}$ Shirley Groer, PhD $;^{2}$ Heather G. Belanger, PhD ${ }^{1-2,4-5}$ \\ ${ }^{1}$ Department of Mental Health and Behavioral Sciences and ${ }^{2}$ Health Sciences Research and Development (R\&D)/ \\ Rehabilitation R\&D Center of Excellence: Maximizing Rehabilitation Outcomes, James A. Haley Veterans' Hospital, \\ Tampa, FL; Departments of ${ }^{3}$ Psychiatry and Neurosciences and ${ }^{4}$ Psychology, University of South Florida, Tampa, FL; \\ ${ }^{5}$ Defense and Veterans Brain Injury Center, Tampa, FL
}

\begin{abstract}
Identification of a remote traumatic brain injury (TBI), particularly mild TBI, is a challenge. The acknowledged standard for determining a history of prior TBI is self-report elicited through a structured or in-depth clinical interview. In April 2007, the Veterans Health Administration (VHA) mandated that the four-section TBI Clinical Reminder screening instrument be completed on all individuals returning from deployment in the Operation Iraqi Freedom/Operation Enduring Freedom theaters of operation (VHA Directive 2007-013). If positive, a follow-up Second Level TBI Evaluation is to be completed. For validation studies of the TBI Clinical Reminder screening process and with the long-term goal of providing a structured methodology to complete the TBI history portion of the Second Level TBI Evaluation, we sought to develop a "criterion standard" semistructured clinical TBI identification interview. This tool was developed through consultation with TBI subject matter experts and built on the strengths of existing tools in the literature. This article describes the six-step developmental methodology and presents the resulting semistructured interview and accompanying manual.
\end{abstract}

Key words: brain injury, clinical reminder, concussion, diagnostic techniques, differential diagnosis, head injury, head trauma, interview, postconcussion syndrome, rehabilitation.

\section{INTRODUCTION}

Evaluating military servicemembers and veterans for possible undetected injuries or conditions of war has been a high priority for the Department of Defense (DOD) and Department of Veterans Affairs (VA). One such condition is traumatic brain injury (TBI), particularly mild TBI. In the context of returning Active Duty servicemembers or veterans presenting for healthcare months or years after a possible mild TBI, the identification of mild TBI is a challenge because of the necessary reliance on subjective report of a relatively remote historical event.

The need to identify and appropriately treat individuals with TBI led the Veterans Health Administration (VHA) to establish a task force to develop clinically appropriate screening and evaluation tools for TBI. Lacking an existing and validated screening instrument, the

Abbreviations: CPRS $=$ computerized patient record system, DOD $=$ Department of Defense, HSR\&D $=$ Health Sciences Research and Development, OIF $/ \mathrm{OEF}=$ Operation Iraqi Freedom/Operation Enduring Freedom, OSU TBI-ID = Ohio State University Traumatic Brain Injury Identification Method, PTSD $=$ posttraumatic stress disorder, TBI $=$ traumatic brain injury, VA = Department of Veterans Affairs, VHA = Veterans Health Administration.

*Address all correspondence to Rodney D. Vanderploeg, PhD; James A. Haley Veterans' Hospital, Mental Health and Behavioral Sciences, Psychology (116B), 13000 Bruce B. Downs Ave, Tampa, FL 33612; 813-972-2000, ext 6728; fax: 813-903-4814. Email: Rodney.Vanderploeg@va.gov http://dx.doi.org/10.1682/JRRD.2011.04.0069 
task force reviewed the literature on the natural history of TBI and existing screening tools and collaborated with the Defense and Veterans Brain Injury Center to develop a TBI screening instrument.

In April 2007, the four-section TBI screening instrument was mandated for all individuals returning from deployment in the Operation Iraqi Freedom/Operation Enduring Freedom (OIF/OEF) theaters of operation (VHA Directive 2007-013). The screening process is initiated through an automated computerized patient record system (CPRS) that triggers a four-section TBI screen, the TBI Clinical Reminder. Patients who respond affirmatively to each of the four TBI screening sections are considered to have a positive screen and are referred for further evaluation. The TBI Clinical Reminder screen was designed to be sensitive to symptomatic individuals who likely sustained a TBI during OIF/OEF deployment.

Hoge et al. [1] and Tanielian and Jaycox [2] have noted high levels of comorbidity and overlap in symptoms across diagnostic conditions in this patient population (e.g., TBI, posttraumatic stress disorder [PTSD], and depression) that may trigger false-positive responses to the TBI screen. Hoge et al. suggested that implementation of such a TBI screening process could have unintended iatrogenic consequences [1].

Although the VHA screening process has been in effect since April 2007, the overall validity (false positives and false negatives) and reliability of the TBI Clinical Reminder are just beginning to be evaluated. However, a recent prospective study of 500 veterans across six VA facilities in one Veterans Integrated Service Network found that the TBI Clinical Reminder had high internal consistency (0.77), test-retest reliability $(0.80)$, and sensitivity $(0.94)$, but only moderate specificity (0.59) [3], consistent with the concerns about excessive false-positive responses. The study utilized research assistants specifically trained to identify TBI by using a comprehensive structured interview. As a result, these reliability estimates are likely inflated compared with the VHA national average. Another test-retest reliability study found relatively poor stability coefficients for individual subitems within the TBI Clinical Reminder (kappa values generally less than 0.50 ) but did not report on the overall test-retest reliability for the screener as a whole (i.e., positive vs negative screen across test-retest) or attempt to address issues of sensitivity and specificity [4].

Following a positive TBI Clinical Reminder screen, VHA clinical programs complete a Second Level TBI
Evaluation by using a defined protocol that includes a clinical history, review of systems, targeted neurological evaluation, the Neurobehavioral Symptom Inventory [5], and an individualized plan of care. A CPRS template was released to the field on October 1, 2007, to facilitate and standardize this evaluation and treatment plan development. However, the template does not provide a structured format for completing a history or determining whether or not a TBI occurred during deployment. Thus, although the template asks clinicians to determine whether or not the "history and clinical course are consistent with a diagnosis of TBI," no methodology is provided to assist with this determination.

From a clinical perspective, determination of whether or not a mild TBI actually occurred is important in differential diagnosis of the current clinical presentation when a past TBI is suspected. Postconcussion symptoms such as problems with attention, memory, irritability, and mood overlap with a variety of mental health conditions, including PTSD and depression. However, if a TBI can be ruled out, then a more valid attribution of symptoms to other causes can be made. From a healthcare system and scientific perspective, accurate determination of whether a mild TBI occurred is essential in the evaluation of outcomes, particularly long-term outcomes. Clearly defined groups (e.g., mild TBI, PTSD, and mild TBI plus PTSD) are crucial for evaluating differential or interactive outcomes or even for developing and evaluating potential biomarkers for remote mild TBI.

Currently, no definitive biomarkers, neuroimaging procedures, or objective clinical tests (e.g., neuropsychological testing or laboratory tests) can determine whether a historically remote event resulted in a mild TBI. More than 80 percent of mild TBIs have no findings on currently available standard clinical neuroimaging procedures [5], although a variety of magnetic resonance imaging techniques, such as diffusion tensor imaging or diffusion kurtosis imaging, show promise [5-6]. The current nonimaging biomarkers that have been identified as having the potential to identify TBI are not specific to TBI and are generally present only in the acute phase and only following moderate to severe TBI [7-8]. Similarly, performance on objective neuropsychological tests is typically normal within 7-30 d following a mild TBI [9] and, in the postdeployed veteran population, is more likely to be adversely affected by deployment stress [10] or PTSD [11-12] rather than mild TBI. 
Without definitive biomarkers, medical observation and documentation of the event, or findings from an immediate medical examination, determining whether or not a historically remote mild TBI occurred depends on self-report elicited through a structured or in-depth clinical interview [13-15]. This process is different from standard diagnostic interviewing, in which current symptoms or laboratory findings are the foundation for making the diagnosis. As will be discussed subsequently, unlike interviewing for other diagnostic conditions, during interviewing for mild TBI, particularly in military, compensation/ pension, or medical-legal settings, a number of issues related to valid assessment arise. These include (1) words used to elicit self-report (e.g., head injury, TBI, concussion, knocked out, loss of consciousness, dazed and confused) are not universally understood and may mean different things to different respondents; (2) memory for details of remote events may be inaccurate for a variety of reasons; (3) issues of secondary gain, such as disability benefits or avoiding military redeployment on the one hand and desires to continue work and career on the other, may influence reporting; and (4) linkage of acute and subsequent symptoms to TBI versus other concomitant causes [16].

For the purposes of further validation studies of the VA TBI Clinical Reminder, particularly determination of its sensitivity and specificity, and with the long-term goal of potentially providing a structured methodology to complete the TBI history portion of the Second Level TBI Evaluation, we sought to develop a "criterion standard" semistructured clinical TBI identification interview building on existing tools in the literature. This article describes the six-step initial developmental methodology and presents the resulting semistructured interview (Appendix 1, available online only) and accompanying manual (Appendix 2, available online only).

\section{METHODS AND RESULTS}

\section{Step 1: Clarify Target or Targets of Interview: What is Meant by TBI?}

The first step of interview development was to review the existing literature on identification of TBIs via interviews and determine what exactly any developed structured interview would need to accomplish.

The general definition of TBI is widely accepted, and the DOD/VHA collaborated on accepting a TBI defini- tion consistent with other definitions of TBI, such as the 1993 definition of the American Congress of Rehabilitation Medicine. These definitions have in common that TBI is a traumatically induced structural injury and/or physiological disruption of brain function as a result of an external force that is indicated by new onset or worsening of at least one of the following clinical signs immediately following the event:

- Any period of loss or decreased level of consciousness.

- Any loss of memory for events immediately before or after the injury.

- Any alteration in mental state at the time of the injury (e.g., confusion, disorientation, slowed thinking).

- Neurological deficits (e.g., weakness, balance disturbance, apraxia, paresis/plegia, change in vision, other sensory alterations, aphasia) that may or may not be transient.

- Intracranial abnormalities (e.g., contusions, diffuse axonal injury, hemorrhages, aneurysms).

In group discussions, we decided that this definition would be used for the current study. We further agreed that at the mild end of the TBI severity spectrum, determination of whether a historically remote event resulted in a TBI is difficult to establish. The minimal criterion for TBI is "any alteration in mental state at the time of the injury." This "alteration of consciousness" is difficult to determine in the combat situation because of the many possible confounding factors and because many deployment-related mild TBIs may be reported. In such situations, all individuals would be likely to experience an adrenalin rush and a "fight-or-flight" response, during which their consciousness is altered and they may well feel dazed or confused during ensuing combat. If asked about alteration of consciousness or feeling dazed and confused following such a combat-related trauma event, most individuals would respond affirmatively, regardless of whether they actually sustained a concussion (i.e., a physiological disruption of brain functioning). This typical psychological response and resulting alteration of consciousness does not reflect a physiological disruption of brain functioning and would not meet the above definition of TBI. Through discussions, we determined that the final interview tool would need to distinguish between these two types of alteration of consciousness: psychological reaction versus physiological disruption of brain functioning.

In this phase of the interview tool development, in addition to the presence of a past TBI event, we discussed 
whether or not the semistructured interview should also clarify the severity of the most severe TBI and attempt to determine the total number of past TBIs sustained. We decided to address these issues in step 4 with the panel of interdisciplinary, nationally recognized TBI experts.

\section{Step 2: Review and Critique Existing TBI Identifica- tion Tools and Integrate Best Aspects of Each}

\section{Review}

Three existing semistructured TBI identification tools were examined:

1. Ohio State University TBI Identification Method (OSU TBI-ID) [15].

2. Structured Interview for TBI Diagnosis, a tool developed by Kerry Donnelly, PhD, at the VA Western New York Healthcare System-Buffalo for use in a VA service-directed grant (Health Sciences Research and Development [HSR\&D] SDR 06-162: “Cognitive Assessment of Veterans after Traumatic Brain Injury"; initial findings published in Donnelly et al., 2011 [3]).

3. Post-Acute Concussion Structured Interview, an unpublished tool developed by William Walker, MD, and colleagues for use in a DOD-funded study (CDMRP W91ZSQ8118N6200001: "Epidemiological Study of Mild Traumatic Brain Injury Sequelae Caused by Blast Exposure during Operations Iraq Freedom and Enduring Freedom").

Corrigan and Bogner [16] cite and concur with the Centers for Disease Control and Prevention that a potential problem with self-report is that the words used to elicit self-report (e.g., head injury, TBI, concussion, knocked out, loss of consciousness, dazed and confused) are not universally understood and may mean different things to different respondents. In addition, these authors point out potential problems with (1) how to elicit memory for remote injuries, (2) how to link neurological symptoms to TBI versus other concomitant causes, and (3) the limits of self-report (e.g., over- or underreporting because of issues such as poor recall, stigma, or secondary gain).

The OSU TBI-ID is an instrument that was created in an attempt to avoid biases due to differences in terminology. It begins by first eliciting recall of all past injuries that received medical attention. Then, it subsequently asks more about those injuries involving a blow to the head or neck or high-velocity forces. Therefore, step 1 (injurious event) of the OSU TBI-ID asks within a speci- fied time frame (e.g., "in the last 3 months" or "in the past year"), "Have you had an injury for which you received medical attention or should have?" and follows with, "Were you drinking or using other drugs at the time of the injury?" Step 2 (TBI criteria) asks, "Were you knocked out or did you lose consciousness?" or "Did the injury cause you to become dazed or confused, or to forget what happened?" A follow-up within step 2 addresses the issue of symptom course and functional consequences of TBI. Questions are asked about "hospitalization or other treatment" and "problems caused by the injury that you did not have before or that got much worse." This set of questions constitutes the interview process.

The Donnelly Structured Interview for TBI Diagnosis is similar to the OSU TBI-ID in a number of respects. For example, it focuses the interview on a specific time frame. However, it begins by asking explicitly about head injury: "I'd like you to think about the most significant head injury you had during your [OIF/OEF] service." It then directly asks about loss or alteration of consciousness: "Did you lose consciousness?" or "Were you disoriented or confused after the event?" It then asks, "What happened leading up to the event?" and "What happened during the event itself?" The interview script ends by asking about immediate symptoms and treatment related to the event.

The Walker Post-Acute Concussion Structured Interview begins with identifying a significant physically traumatic event and then asks an open-ended question: "You indicated that on [date] you experienced a [type of accident/event/trauma]. Please tell me in as much detail as possible what happened." This provides a forum for the respondent to provide a detailed narrative about events leading up to the trauma; information about the event; and information about what happened after the accident, including what was experienced physically and emotionally. The script then asks directly about "memory gaps" before or after the event. Then respondents are asked about (1) the mechanism of injury; (2) "loss of consciousness" but defined by "that is, you could not see, speak, and move for some period of time"; and (3) immediate symptoms, such as feeling dazed or confused, seeing stars, feeling dizzy or irritable, hearing loss, blindness, headache, abdominal or stomach pain, and any other feelings or problems. 


\section{Critique}

Note that each tool was developed for a specific purpose and/or population. For example, the Donnelly Structured Interview for TBI Diagnosis was developed for a study to serve as the comparison standard to test the VA TBI Clinical Reminder. As a result, the language included in this interview had to parallel the TBI Clinical Reminder, which is why it directly asks about the loss or alteration of consciousness. Such differences between the existing approaches are to be expected, and each tool may work very well for its intended purpose.

Despite the similarities and strengths of these tools, concerns arose that only the Walker Post-Acute Concussion Structured Interview defines "loss of consciousness" for the interviewee and all three scripted interviews ask about feeling "dazed," "confused," and "disoriented" without defining these constructs for respondents.

Therefore, for the most part, the concerns expressed by Corrigan and Bogner [16] regarding unclear terminology either are not addressed or are only partially addressed by these tools. Also not fully addressed are concerns regarding (1) linking the trauma event to TBIrelated neurological symptoms versus other concomitant causes and (2) over- or underreporting as a result of factors such as stigma or secondary gain. These findings were brought to the expert panel for consideration and discussion as part of step 3 .

\section{Step 3: Convene Panel of Interdisciplinary, Nationally Recognized TBI Experts to Review Integrated Approach and Develop Semistructured Interview Tool Based on Strengths and Extension of Existing Tools and Using Panel Consensus}

A panel of experts in TBI was convened to establish a criterion standard semistructured clinical interview for assessing whether a TBI has occurred. The expert panel consisted of providers who are nationally recognized for their expertise in TBI and care for OIF/OEF veterans with TBI/polytrauma and blast-related injuries. Because many of the individuals administering the TBI Clinical Reminder are medical doctors and psychologists, we limited this expert panel to those professions.

As a starting point for the expert panel meeting, we assembled already developed interviews that are currently being used. These included (1) Corrigan and Bogner's OSU TBI-ID [16], (2) the Donnelly Structured Interview for TBI Diagnosis, (3) the Walker Post-Acute Concussion Structured Interview, and (4) an initial rough draft of the proposed VA TBI Identification Interview. All members of the expert panel were sent the documents in advance of the actual meeting.

On the day of the meeting, an expert moderator of focus groups and qualitative research stated the objective of the panel session to ensure that everyone understood the tasks and expected outcomes. Then, the existing TBI interviews were presented and the panel was asked to consider the strengths and limitations of each tool. The panel was also asked to consider whether the new tool should address the number of TBIs, the etiology of the TBI, and the severity of the TBI. After the discussion, it was decided that the patients should narrate their own story, and on the basis of the evidence provided, the interviewer would make a decision regarding the occurrence of a TBI. This is a similar format to the Walker PostAcute Concussion Structured Interview. It was also decided that the most severe event should be considered. After completion of the interview for the most severe event, additional information could be collected regarding the number of TBIs. As a result, the panel decided that key elements from each of the reviewed tools would be combined for the creation of the new tool.

The expert panel also recommended that material be developed for subsequent training of clinicians (such as a training manual and videos showing the process) if the tool is later implemented on a large-scale basis. Some additional discussion concerned how the tool could be implemented in the VA.

The structured interview based on the expert panel recommendations consisted of three parts with the recording form arranged in three parallel columns:

1. Part A is a series of open-ended questions (and follow-up probes) to (a) facilitate the patient's freely told, spontaneous description of the event, (b) help determine whether the event resulted in a concussion or TBI, (c) determine the cause of the physical trauma (e.g., motor vehicle accident, fall, explosive blast), (d) identify any immediate new onset symptoms or problems, and (e) determine the course of any new symptoms.

2. Part B is a checklist form for recording the patient's spontaneously reported information from part A's semistructured interview.

3. Part $\mathrm{C}$, the final part, is a series of questions and recording form for confirming the patient's responses acquired during part $\mathrm{A}$ and recorded on the part $\mathrm{B}$ form. This includes confirming the negative if the 
interviewee did not report an event with alteration of consciousness and/or did not report experiencing acute onset or worsening of new symptoms. For example, regarding symptoms, the confirmation would include asking, "Let me see if I understood you correctly about any symptoms/problems you experienced after the event. You indicated that you had no worsening problems with headaches, memory, dizziness, irritability, light sensitivity, or sleep after this event, correct?"

The interview form then asks the examiner, if he or she determines that a TBI likely occurred based on the responses to parts $\mathrm{A}-\mathrm{C}$, to ask questions about the (1) number of events that likely met criteria for a TBI, (2) dates of the worst and most recent such TBIs (if more than one), and (3) severity of the worst and most recent such TBIs (if more than one). The form ends with asking the examiner to rate the severity of the worst and most recent TBIs (if such occurred) on an 8-point scale and to offer a final rating of the likelihood that a TBI occurred.

\section{Step 4: Send Modified Interview Tool and Accompa- nying Manual Back to Expert Panel for Review and Refinement}

Two documents were sent back to the expert panel for review and refinement. These included the new VA TBI Identification Clinical Interview and an accompanying manual, the latter of which (1) described the semistructured interview approach, (2) defined TBI, (3) defined other terms relevant to the interviewer (e.g., dazed, confused, disoriented, loss of consciousness, coma) and differentiated them from psychological trauma responses, and (4) described the natural history of a mild TBI or concussion. Documents were refined based on the comments received.

\section{Step 5: Refine and Modify Interview Tool and Accom- panying Manual Based on Clinicians Using Them Clinically in Field}

Next, the VA TBI Identification Clinical Interview and accompanying manual were used for several weeks in three different TBI clinical settings throughout the VA (i.e., Tampa; Minneapolis; and Washington, DC). Feedback following use was focused primarily on the layout of the interview administration and recording tool and on whether or not each question and symptom needed to be asked or checked. As a result, the layout of the interview/ recording tool was modified into a bifold (Figure). In addition, the accompanying manual (p. 4 of Appendix 2) was modified to clarify that-

Interview questions and follow-up probes are designed to help question the patient. The purpose is to gather information that will allow the examiner to determine whether or not a TBI has occurred. It is not essential that every question be asked, but rather the questions provide a means to query the patient about an event that may or may not have resulted in a TBI.

If patients describe the potential TBI event in detail, no additional questions will need to be asked. However, follow-up questions are provided as prompts or cues to help guide the patient to provide the needed information. However, patients are never asked directly about:

- TBI or TBI-related conditions,

- Loss of consciousness; memory gaps; or being dazed, confused or disoriented, or

- Specific symptoms.

\section{Step 6: Re-Examine and Finalize Clinician-Modified Interview Tool and Manual by Panel of Interdisciplin- ary TBI Experts}

The only additional refinements made at this step were to the final classification descriptors for severity of any likely TBIs. It was felt that, in some cases, it would not be possible to determine whether an individual actually lost consciousness for a brief period of time or had no memory for a period of time but was not actually unconscious. Thus, the phrasing was changed for the four lowest severity rankings from "no loss of consciousness" of various durations to "no loss or possible loss of consciousness" of various durations (p. 7 and 8 of Appendix 1).

\section{DISCUSSION}

This article describes the development of the VA TBI Identification Clinical Interview. This tool was developed to provide a standardized method for determining whether or not an individual experienced a TBI in the past (within certain prespecified time frames). Development specifically sought to minimize limitations and concerns expressed in the literature regarding (1) nonshared terminology, such that the same terms are not universally understood (e.g., TBI, loss of consciousness, dazed and 


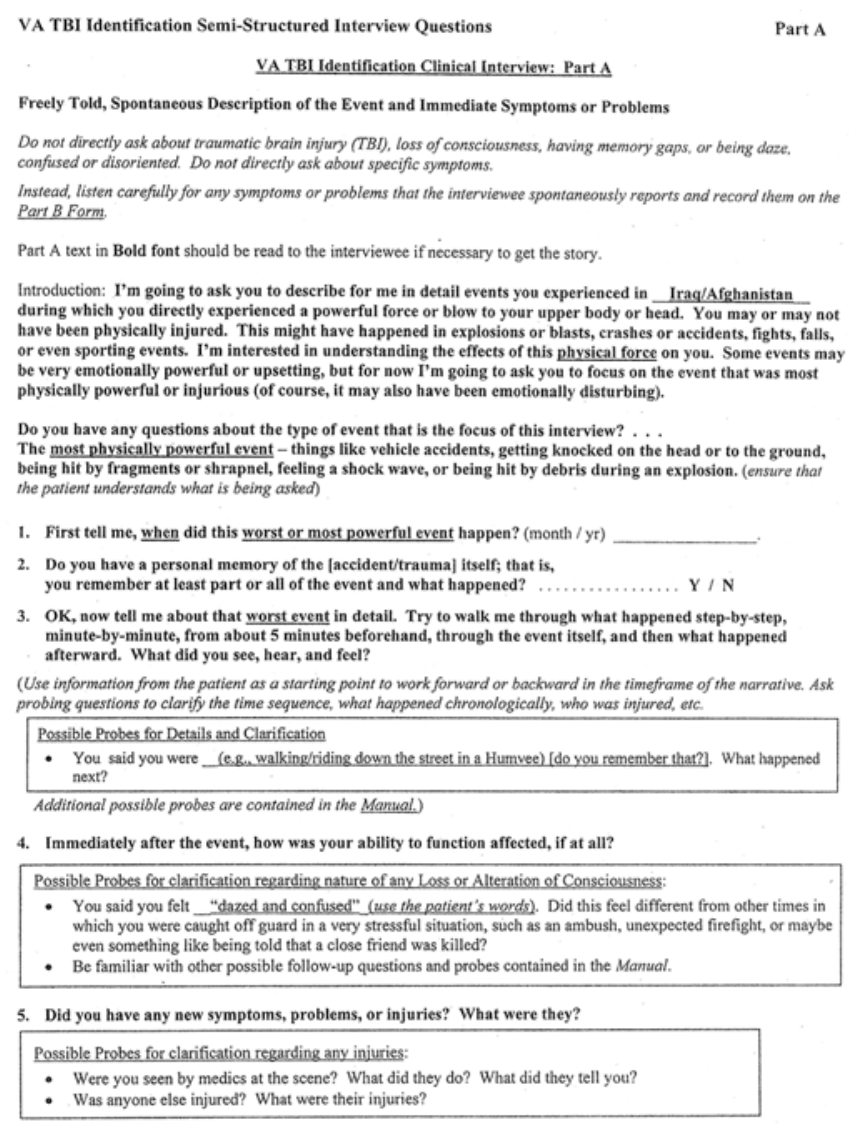

Page 1

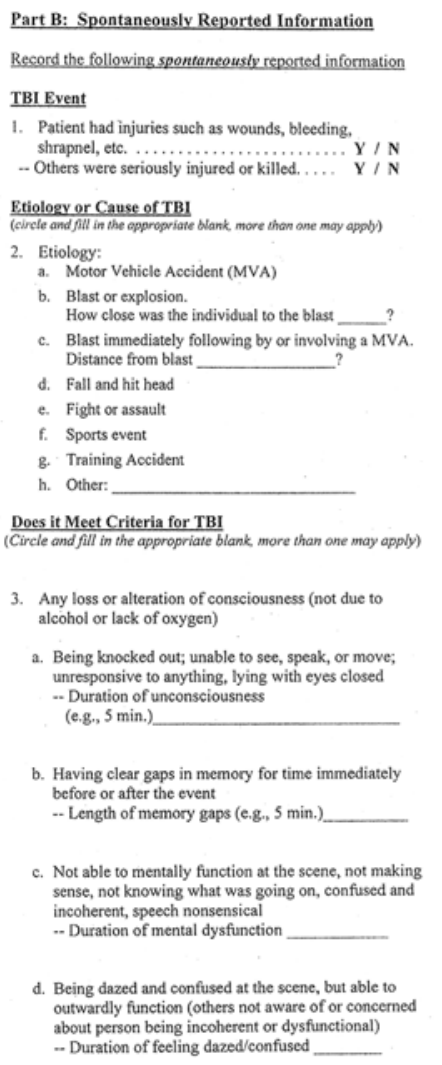

Part C: Follow-up Confirmatory Questions

Now I'm going to ask some additional questions to make sure that I correctly understood what you experienced.

Confirmation of Memory for the Event

1. You said you have a personal memory of the event or at
least part of the event and what happened, correet? $\mathrm{Y} / \mathrm{N}$ least part of the event and what happened,

Confirmation of the Etiology of the Event

2. You said that your injuries were due to-
a. Motor Vehicle Accident (MVA)

b. Blast or explosion, and you were about

feet from the blast?

B. Blast immediately followed by a MVA, and you were

- Blast, immediately followed by a MVA, and you were

d. Fall and hit head

. Fight or assault

f. Sports event

g. Training Accident

h. Other:

\section{Confirmation of TBI (or no TBI)} Confirmation of TBI (or no TBI)
(Confirm all of the items below that fit with the spontantous story:
mare than one can be checked)

3. Let me see if I understood what you experienced right after the event. You indicated that

a. Were knocked out; that is, you could not see, speak, or move \& were unresponsive to anything, lying with eyes chestis correct? $\mathrm{Y} / \mathrm{N}$ -- Were you told that by a witness? ........... $\mathrm{Y} / \mathrm{N}$

b. Had gaps in your memory for
BEFORE the event, is this correct? ............ just Bad gaps in your memory for AFTER the event, is this correet? .......... just

d. Were unable to function in a normal way at the scene. You were not making sense, or did not know what was going on, or were confused and incoherent, and this went on for
event, is this correct? .................... Y Y / N

c. Dazed and confused at the scene for but outwardly appeared to be able to function $\mathrm{OK}$, is this

f. It sounds like there are no holes or gaps in your memory from that day and that you were functioning in a pretty normal way under the circumstances at the scene and
afterward, is that correct? ................ Y / N

Page 2

Figure.

Example of bifold layout of Department of Veterans Affairs (VA) Traumatic Brain Injury (TBI) Identification Clinical Interview.

confused); (2) how to elicit memories for remote events; (3) limits of self-report, particularly in relationship to over- or underreporting as a result of issues such as poor recall, stigma, or secondary gain; and (4) linkage of neurological symptoms to TBI versus other concomitant causes. Each of these issues will be dealt with in turn.

\section{Avoiding Nonshared Terminology}

One method to minimize problems due to nonshared terminology is to provide basic education to the potential interviewer about the definition of TBI, typical post-TBI symptoms and their course, and clarification of what constitutes an alteration of consciousness (e.g., terms such as dazed, confused, disoriented, posttraumatic amnesia, and coma/loss of consciousness). The VA TBI Identification Clinical Interview manual specifically addresses each of these issues and differentiates them from similar symp- toms that may have a psychological origin versus a physiological disruption of brain functioning.

A second method of minimizing problems due to nonshared terminology is to avoid using these terms altogether. Instead of asking about TBI or alteration of consciousness in the VA TBI Identification Clinical Interview, the interviewee is asked to describe in detail events in which "you directly experienced a powerful force or blow to your upper body or head. You may or may not have been physically injured. This might have happened in explosions or blasts, crashes or accidents, fights, falls, or even sporting events. . . Tell me about the worst event in detail. Try to walk me through what happened step-by-step, minute-by-minute, from about 5 minutes beforehand, through the event itself, and then what happened afterward. What did you see, hear, and feel?" Asking about physically powerful events and having 
patients describe in their own words what they experienced - saw, heard, and felt - eliminates problems due to misunderstanding of terminology.

As a final means to ensure mutual understanding, in the VA TBI Identification Clinical Interview, the patient is asked to confirm what the interviewer heard. In this way, a final validity check is provided to ensure that the interviewer did not misinterpret the patient's recall of events or choice of terminology. This can be thought of as "playing the tape recorder back" for the patient to ensure its fidelity. This includes confirming the negative if the interviewee did not have an event with alteration of consciousness and/or did not experience acute onset or worsening of new symptoms.

\section{Eliciting Memories for Remote Events}

Similar to the OSU TBI-ID approach, the VA TBI Identification Clinical Interview provides a context and time frame for consideration. In the OSU TBI-ID, the provided context is "injury for which you received medical attention or should have"; in the Donnelly Structured Interview for TBI Diagnosis, the provided context is "significant head injury you had during your [OIF/OEF] service"; and in the Walker Post-Acute Concussion Structured Interview, the provided context is "experienced a [type of accident/event/trauma]." In the newly developed VA TBI Identification Clinical Interview, the provided context is "directly experienced a powerful force or blow to your upper body or head." In using the VA TBI Identification Clinical Interview, the interviewer does not ask for injuries; rather, the interviewer says, "You may or may not have been physically injured." This opens up the interviewee to describe experiences that may be excluded by the provided contexts of the alternative interviews but that may have indeed resulted in a TBI despite the patient's belief.

All four interviews provide specific time frames for the patient to consider in responding to questions. In other words, patients are not asked to provide answers for their entire life span. Providing specific time frame anchors allows for more accurate retrospective recall (e.g., van der Vaart and Glasner [17]). All but the OSU TBI-ID focus on a period of military deployment, but the OSU TBI-ID can be adapted to initially ask about other potential time frames.

The new VA TBI Identification Clinical Interview attempts to enhance accurate recall by asking about several specific time points after the event-any symptoms noticed at the time of the first meal after the event, that night when going to bed, and the next morning when waking up. Similarly, only the new VA TBI Identification Clinical Interview asks about the course of any symptoms spontaneously reported by the interviewee (i.e., "over the course of the next several days, weeks and months, what happened to these symptoms or problems? When did you feel back to yourself or $100 \%$ ?"). These questions also help to elicit and clarify memories for the event and the acute postevent period.

\section{Minimizing Over- or Underreporting}

Many potential causes of over- or underreporting exist. A person may over- or underreport because of poor recall. In the case of individuals who sustained a TBI in combat, underreporting may occur because of legitimate memory loss about experiences or concerns about how symptoms may affect career issues. Overreporting may occur because of a tendency to be agreeable. In other words, if an interviewer is asking about head injury, the patient may feel compelled to "give them what they want" or to embellish in order to provide a more "useful" story. Overreporting may also occur for secondary gain.

Being directly asked about TBI or head injury makes it clear what a person might wish to over- or underreport. That is, certain demand characteristics are associated with such questions. For example, people may feel that if they are being asked about TBI, then they "should" be forgetful or they "should" have lost consciousness, etc. Demand characteristics have been shown to exert powerful effects on people's behavior in a wide variety of settings by promoting expectancy-consistent changes in behavior (e.g., see Darkes and Goldman, Faith et al., and Nichols and Maner [18-20]).

Of the existing TBI identification interviews, only the new VA TBI Identification Clinical Interview does not directly ask about TBI or other injuries; instead it asks about experiencing powerful physical events. In addition, in the initial data collection phase, only the VA TBI Identification Clinical Interview asks open-ended questions and asks for clarification or more details, but does not ask specifically about TBI, loss of consciousness, disorientation, or other acute symptoms indicative of TBI by using those terms. The interviewer knows that the focus of the interview is TBI, but the interviewees only know that they are being asked to describe what happened to them following a physically powerful event. 
Therefore, the potential for demand characteristics to influence patient responding is decreased.

The VA TBI Identification Clinical Interview confirms the absence of alteration of consciousness, memory gaps, or acute-onset new symptoms. To minimize underreporting, it confirms that none of the following symptoms was experienced after the trauma event: memory gaps, difficulty functioning, headaches, dizziness, irritability, light sensitivity, memory difficulties, or sleep problems after the event. If these symptoms had occurred, providing these examples at this phase of the interview process should provide a memory trigger to assist recall.

\section{Linking Neurological Symptoms to TBI}

Traditionally, medical causality follows a linear model in which a specific event (e.g., infection, injury) leads to symptoms, which are verified by history, medical examination, and diagnostic tests. A diagnosis is established when symptom onset and course are determined to be consistent with one particular etiological explanation and inconsistent with alternative etiologies [21]. In the area of mild TBI, accurate diagnosis depends on several factors. First, a clear traumatic event, potentially severe enough to disrupt brain functioning, must have occurred. Second, the initial clinical presentation following that event must meet the criteria for mild TBI, that is, a physiologic disruption of brain function resulting in a relatively short period of lost or altered consciousness. Third, ongoing or emerging symptoms and their course must be consistent with brain dysfunction and must not be attributable to other etiological factors, e.g., psychological trauma.

In the case of a remote mild TBI, the above factors typically depend on an individual's self-report, which in turn depends on accurate recall and reporting - uncomplicated by over- or underreporting. Potential over- or underreporting can be minimized by using open-ended questions that avoid suggesting "expected" symptoms. Although all four interviews ask about new onset or worsening symptoms immediately after the trauma event, only the Donnelly Structured Interview for TBI Diagnosis and the new VA TBI Identification Clinical Interview avoid asking about any specific set of symptoms. The OSU TBI-ID and the Walker Post-Acute Concussion Structured Interview both ask about specific symptoms immediately after the trauma event.

\section{LIMITATIONS AND FUTURE DEVELOPMENT ISSUES}

This description of the initial development of the VA TBI Identification Clinical Interview represents only the first phase in a line of research that will be necessary to validate this approach and evaluate whether this new interview tool more accurately confirms the occurrence of a remote mild TBI. The patient-narrated story approach to clinical interviewing used in this article is hypothesized to provide a more factual account of the "event" than other approaches or even simply asking the four basic questions composing the current VA TBI Clinical Reminder. Although this approach was based on consensus opinion of TBI experts, this hypothesis will need to be examined in future research.

In this initial developmental process, the expert consensus panel recommended that material be developed for subsequent training of clinicians if the tool is to be implemented on a large-scale basis. However, this recommendation is premature. More work is needed to evaluate the interrater reliability and validity of this new tool. In addition, determination of the presence or absence of a remote mild TBI is only one aspect of the clinical process involved in evaluation and treatment of individuals returning from deployment with a variety of symptoms and concerns. This interview may help with differential diagnosis and potentially with attribution of symptoms to different comorbid conditions. However, beyond the scope of the currently described VA TBI Identification Clinical Interview are additional aspects of the VA's Second Level TBI Evaluation process that will be necessary to develop and implement successful treatments. However, the VA TBI Identification Clinical Interview holds promise because it has attempted to address concerns expressed in the literature [16] regarding existing interview approaches and presents a methodology for better linkage of symptoms to TBI versus other possible causes.

A limitation of all interview approaches is that those veterans who had a TBI and are having memory problems may fail to recall and report events and symptoms accurately. For such individuals, symptom checklists may result in more accurate reporting because they may trigger memory for forgotten symptoms. However, the primary purpose of the VA TBI Identification Clinical Interview is to confirm whether or not an individual sustained a TBI. 


\section{CONCLUSIONS}

The new VA TBI Identification Clinical Interview attempts to build on the strengths of existing TBI identification interviews and, at the same time, address their limitations. It was developed largely with a VA population in mind. The VA TBI Identification Clinical Interview is unique in that it does not ask leading or closed-ended questions, other than in attempting to clarify or confirm what the interviewee reported spontaneously. Interviewees are never asked directly about (1) TBI or TBI-related conditions; (2) loss or alteration of consciousness; memory gaps; or being dazed, confused or disoriented; or (3) any specific symptom or set of symptoms. The interview itself consists of two parts: an open-ended set of questions designed to help interviewees describe what they experienced during and following a physically powerful event, followed by a set of confirmatory questions to clarify interviewees' spontaneous self-report. The confirmatory questions clarify whether the criteria for TBI were met, whether there were new symptoms (or symptoms significantly worse than usual) following the event, the onset of those symptoms over the next several days, and the course of any new symptoms over the subsequent days and weeks.

A rigorous six-step process was undertaken in the development of this new tool. The VA TBI Identification Clinical Interview and accompanying manual were sent to a number of TBI providers at three different VA facilities. Preliminary feedback from clinicians using this tool has been positive. Clinicians report that the interview may be completed in 15 to $20 \mathrm{~min}$ and that they are able to use the recording form to gather the information and address the relevant issues. Future work should examine the reliability and validity of this interview process.

\section{ACKNOWLEDGMENTS}

\author{
Author Contributions: \\ Study concept and design: R. D. Vanderploeg, S. Groer, \\ H. G. Belanger. \\ Acquisition of data: R. D. Vanderploeg, S. Groer. \\ Analysis and interpretation of data: R. D. Vanderploeg, S. Groer. \\ Drafting of manuscript: R. D. Vanderploeg. \\ Critical revision of manuscript for important intellectual content: \\ R. D. Vanderploeg, S. Groer, H. G. Belanger. \\ Obtained funding: R. D. Vanderploeg, S. Groer. \\ Administrative, technical, or material support: R. D. Vanderploeg, \\ S. Groer.
}

Study supervision: R. D. Vanderploeg, S. Groer.

Financial Disclosures: The authors have declared that no competing interests exist.

Funding/Support: This material was based on work supported by the VA and VHA (grant SDR HSR\&D 08-411- TBI Screening Instruments and Processes) and the James A. Haley Veterans' Hospital. Additional Contributions: The authors acknowledge the expert panel members: Heather G. Belanger, PhD; Kerry T. Donnelly, PhD; Henry L. Lew, MD; Nina A. Sayer, PhD; Jennifer J. Vasterling, PhD; Marina Waisman, MD; and William C. Walker, MD. The authors also acknowledge Melody Schiaffino, MPH, and Gail Powell-Cope, PhD, ARNP, FAAN, for their work on this project.

Disclaimer: The views expressed herein are those of the authors and do not necessarily reflect the views or the official policy of the VA or U.S. Government.

\section{REFERENCES}

1. Hoge CW, McGurk D, Thomas JL, Cox AL, Engel CC, Castro CA. Mild traumatic brain injury in U.S. Soldiers returning from Iraq. N Engl J Med. 2008;358(5):453-63. [PMID: 18234750] http://dx.doi.org/10.1056/NEJMoa072972

2. Tanielian TL, Jaycox LH. Invisible wounds of war: psychological and cognitive injuries, their consequences and services to assist recovery. Santa Monica (CA): RAND; 2008.

3. Donnelly KT, Donnelly JP, Dunnam M, Warner GC, Kittleson CJ, Constance JE, Bradshaw CB, Alt M. Reliability, sensitivity, and specificity of the VA traumatic brain injury screening tool. J Head Trauma Rehabil. 2011;26(6):439-53. [PMID:21386716] http://dx.doi.org/10.1097/HTR.0b013e3182005de3

4. Van Dyke SA, Axelrod BN, Schutte C. Test-retest reliability of the Traumatic Brain Injury Screening Instrument. Mil Med. 2010;175(12):947-49. [PMID:21265299]

5. Belanger HG, Vanderploeg RD, Curtiss G, Warden DL. Recent neuroimaging techniques in mild traumatic brain injury. J Neuropsychiatry Clin Neurosci. 2007;19(1):5-20. [PMID:17308222] http://dx.doi.org/10.1176/appi.neuropsych.19.1.5

6. Grossman EJ, Ge Y, Jensen JH, Babb JS, Miles L, Reaume J, Silver JM, Grossman RI, Inglese M. Thalamus and cognitive impairment in mild traumatic brain injury: a diffusional kurtosis imaging study. J Neurotrauma. Forthcoming 2011. [PMID:21639753] http://dx.doi.org/10.1089/neu.2011.1763

7. Iverson GI, Lange R, Gaetz M, Zasler N. Mild traumatic brain injury. In: Zasler N, Katz D, Zafonte R, editors. Brain injury medicine: principles and practice. New York (NY): Demos Medical Publishing; 2006.

8. Mussack T, Dvorak J, Graf-Baumann T, Jochum M. Serum $\mathrm{S}-100 \mathrm{~B}$ protein levels in young amateur soccer players 
after controlled heading and normal exercise. Eur J Med Res. 2003;8(10):457-64. [PMID:14594652]

9. Belanger HG, Curtiss G, Demery JA, Lebowitz BK, Vanderploeg RD. Factors moderating neuropsychological outcomes following mild traumatic brain injury: a metaanalysis. J Int Neuropsychol Soc. 2005;11(3):215-27.

[PMID:15892898] http://dx.doi.org/10.1017/S1355617705050277

10. Vasterling JJ, Proctor SP, Amoroso P, Kane R, Heeren T, White RF. Neuropsychological outcomes of army personnel following deployment to the Iraq war. JAMA. 2006;296(5):519-29. [PMID:16882958] http://dx.doi.org/10.1001/jama.296.5.519

11. Cooper DB, Mercado J, Critchfield E, Kennedy J, Gaylord KM. Cognitive functioning following explosion injuries in OIF/OEF service members: relationship to trauma severity, mild traumatic brain injury, and psychiatric diagnosis. Arch Clin Neuropsychol. 2008;23:731-32.

12. Mercado-Couch JM, Cooper DB, Critchfield E, Kennedy J, Gaylord KM. Screening for cognitive dysfunction in OIF/ OEF service members with explosion injuries admitted to a burn unit. Arch Clin Neuropsychol. 2008;23:731.

13. Bogner J, Corrigan JD. Reliability and predictive validity of the Ohio State University TBI identification method with prisoners. J Head Trauma Rehabil. 2009;24(4):279-91. [PMID:19625867] http://dx.doi.org/10.1097/HTR.0b013e3181a66356

14. Diamond PM, Harzke AJ, Magaletta PR, Cummins AG, Frankowski R. Screening for traumatic brain injury in an offender sample: a first look at the reliability and validity of the Traumatic Brain Injury Questionnaire. J Head Trauma Rehabil. 2007;22(6):330-38. [PMID:18025965] http://dx.doi.org/10.1097/01.HTR.0000300228.05867.5c

15. Schwab KA, Ivins B, Cramer G, Johnson W, Sluss-Tiller M, Kiley K, Lux W, Warden D. Screening for traumatic brain injury in troops returning from deployment in Afghanistan and Iraq: initial investigation of the usefulness of a short screening tool for traumatic brain injury. J Head Trauma Rehabil. 2007;22(6):377-89. [PMID:18025970] http://dx.doi.org/10.1097/01.HTR.0000300233.98242.87

16. Corrigan JD, Bogner J. Initial reliability and validity of the Ohio State University TBI Identification Method. J Head
Trauma Rehabil. 2007;22(6):318-29. [PMID:18025964] http://dx.doi.org/10.1097/01.HTR.0000300227.67748.77

17. van der Vaart W, Glasner T. Applying a timeline as a recall aid in a telephone survey: a record check study. Appl Cogn Psychol. 2007;21(2):227-38. http://dx.doi.org/10.1002/acp.1338

18. Darkes J, Goldman MS. Expectancy challenge and drinking reduction: experimental evidence for a mediational process. J Consult Clin Psychol. 1993;61(2):344-53. [PMID:8473588] http://dx.doi.org/10.1037/0022-006X.61.2.344

19. Faith MS, Wong FY, Allison DB. Demand characteristics of the research setting can influence indexes of negative affect-induced eating in obese individuals. Obes Res. 1998; 6(2):134-36. [PMID:9545020]

20. Nichols AL, Maner JK. The good-subject effect: investigating participant demand characteristics. J Gen Psychol. 2008; 135(2):151-65. [PMID:18507315] http://dx.doi.org/10.3200/GENP.135.2.151-166

21. Vanderploeg RD, Belanger HG, Curtiss G. Mild traumatic brain injury: neuropsychological causality modeling. In: Young G, Kane A, Nicholson K, editors. Psychological knowledge in court: PTSD, pain and TBI. New York (NY): Springer-Verlag; 2006. p. 279-307.

Submitted for publication April 7, 2011. Accepted in revised form October 19, 2011.

This article and any supplementary material should be cited as follows:

Vanderploeg RD, Groer S, Belanger HG. Initial developmental process of a VA semistructured clinical interview for TBI identification. J Rehabil Res Dev. 2012;49(4): 545-56.

http://dx.doi.org/10.1682/JRRD.2011.04.0069

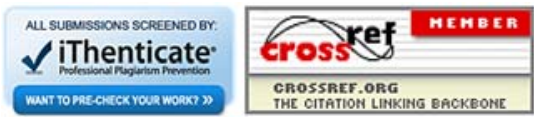


